\title{
Effective and Efficient Tracking and Ego-Motion Recovery for Mobile Cameras
}

\author{
Huiyu Zhou ${ }^{1}$ and Gerald Schaefer ${ }^{2}$ \\ 1 Institute of Electronics, Communications and Information Technology \\ Queen's University Belfast, Belfast, U.K. \\ 2 Department of Computer Science \\ Loughborough University, Loughborough, United Kingdom
}

\begin{abstract}
Estimating 3-D structure and camera motion from 2-D image sequences is an important problem in computer vision. In this paper we present an effective approach to tracking and recovery of ego-motion from an image sequence acquired by a single camera attached to a pedestrian. Our approach consists of two stages. In the first phase, human gait analysis is performed and human gait parameters are estimated by frame-by-frame analysis utilising a generalised least squares technique. In the second phase, the gait model is employed within a "predict-correct" framework using a maximum a posteriori expectation maximisation strategy to recover ego-motion and scene structure, while continuously refining the gait model. Experiments on synthetic and real image sequences confirm that the use of the gait model allows for effective tracking while also reducing the computational complexity.
\end{abstract}

\section{Introduction}

Estimating 3-D structure and camera motion from 2-D images is one of the fundamental problems in computer vision. One strategy for recovering structure from passive image data is structure from motion (SFM) which also allows recovery of the ego-motion of the sensor with respect to a world co-ordinate system, performing 3-D scene reconstruction and navigation [1]. The majority of existing methods for feature tracking use frame-to-frame prediction models based for example on Kalman filters [2], particle filtering [3], or optimisation based approaches [4].

Although working well in certain applications, these approaches do not take into account the long-term history of the camera's motion. In contrast, in this paper we track features and recover ego-motion from a temporal image sequence acquired by a single camera mounted on a moving pedestrian. We show that the use of an explicit longer term, non-linear human gait model is more efficient in this case since fewer features are lost and the processing time per frame is reduced as either the search window or the frame rate can be reduced. Our work is inspired by the study of Molton et al. [5] who used a robotic system to measure the gait of a walking person, while a digital compass and an inclinometer were used to record rotation. They employed an iterated and extended Kalman filter to initialise wavelet parameter estimates, then running across the whole period of activity. In our work, motion is computed directly from video data, and the emphasis is on the use of a longer term model to increase algorithmic efficiency.

S. Chaudhury et al. (Eds.): PReMI 2009, LNCS 5909, pp. 345-350. 2009.

(C) Springer-Verlag Berlin Heidelberg 2009 


\section{Tracking and Ego-Motion Recovery}

In the following, we use the term "ego-motion" to refer to both frame-to-frame and longer-term periodic motion while "camera transformation" refers to the ego-motion of the camera between any two frames, and "gait model" to the longer-term ego-motion of the camera over many frames. Our proposed approach consists of two phases: initialisation and continuous tracking. We assume that $m$ frames are included in the initialisation phase and $n$ frames $(n>m)$ in the whole sequence, and that the intrinsic camera parameters are known.

\subsection{Phase 1: Initialisation}

The purpose of the first phase is to acquire the long-term gait model of the pedestrian. The general workflow of the initialisation phase is outlined in Algorithm 1 Of the well-tested feature tracking algorithms, reviewed by Lepetit and Fua [6], the ShiTomase-Kanade (STK) tracker [7] is chosen because of its accuracy and reliability. The STK tracker has two stages: feature selection and feature tracking. The feature selection process computes the eigenvalues of a gradient function for each pixel, comparing the result with a fixed threshold. In the published algorithm, image features with higher eigenvalues are considered as good features to track. However, in our approach we use the SUSAN (Smallest Univalue Segment Assimilating Nucleus) [8] operator instead to select an initial feature set in the first frame, due to its known robustness to noise. This is not used subsequently, unless the number of tracked features falls below a pre-defined threshold in which case we re-initialise with new features.

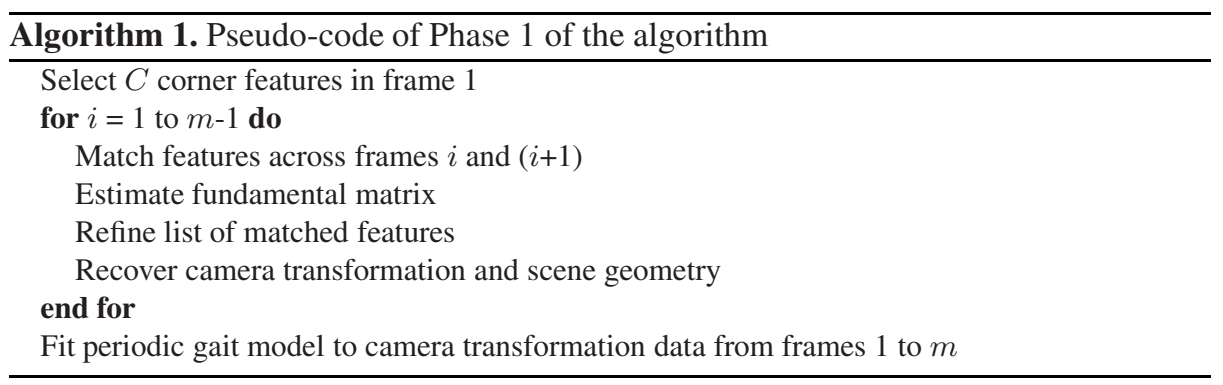

As we track features in successive frames, we minimise the residual error using an affine transformation, as in the STK algorithm, assuming the displacement is small. However, we use a generalised least squares (GLS) algorithm [9] to recover the frameto-frame camera transformation and scene geometry. The application of GLS provides a more robust estimation technique that can derive good results also when the error distribution is not normally distributed [10]. Typically, $m$ is chosen large enough to recover about two complete strides, e.g. $m=50$ for a $25 \mathrm{~Hz}$ sampling rate. The number of features $C$ is a user parameter, typically set to 150 in our experiments. This leads to the recovery of a temporary motion model with 6 degrees of freedom, i.e. the 3 displacements and 3 Euler angles, which can be stored in a gait library using a truncated Fourier series representation. 


\subsection{Phase 2: Continuous Tracking}

In Phase 2, outlined in Algorithm 2, we use and update the gait model to improve the prediction of the location of features in each new frame. Since this prediction is based on a longer history, not every frame needs to be considered and we include a parameter $k$ that describes the gap between frames. The coarse-to-fine matching parameters, e.g. window size, are selected according to the variance of the Euclidean distance between the predicted and measured feature positions. A maximum a posteriori expectation maximisation (MAP-EM) algorithm is used to find the best feature match and determine the camera transformation parameters between two frames. The gait model is examined and updated if necessary on a periodic basis using iteratively re-weighted least squares (IRLS) [11]. To maintain its validity, the $m$ most recent frames are used, applying Spearman correlation to compare the recent gait parameters individually with the stored values. If the correlation coefficients are lower than a pre-defined threshold (typically 0.9), then the current gait model is updated. Accumulation of errors in the motion tracking can deteriorate the performance of the proposed tracker. In order to reduce this accumulated error we can re-estimate the overall parameters by collecting very similar (or the same) images that were just used. For example, multiple estimates of the position of a known 3-D point should be identical. This strategy works effectively if the iteration runs several times.

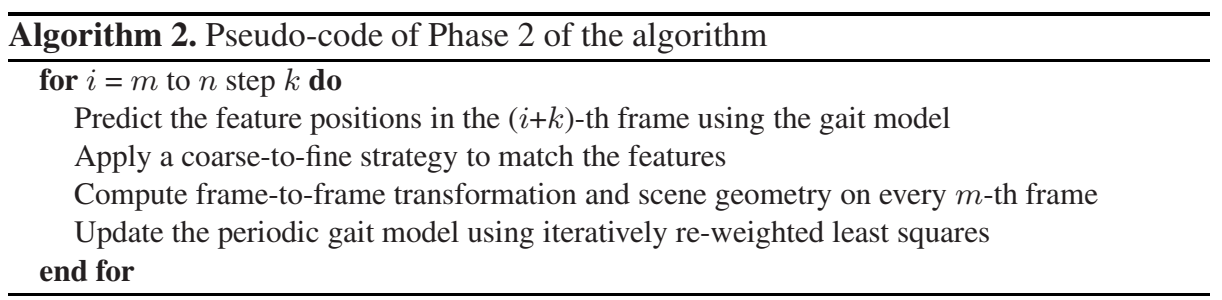

\section{Experimental Results}

To demonstrate improved performance, we compare the proposed gait-based egomotion tracking with the original STK algorithm which uses a short-term displacement model. Experiments were obtained based on several synthetic and real image sequences, however due to space limitation we present here only the results of one synthetic and one real sequence.

The synthetic test data is obtained from a computer game simulation 11 for comparison of the algorithms with known periodic gait parameters. We can compare the recovered parameters against the ground truth as the gait parameters can be fully programmed using the game engine. Here we show the results of a demonstration video ("rolling sequence") in which the player wanders in a castle in changing illumination, which together with the more complex 3-D environment can cause missed feature

\footnotetext{
${ }^{1}$ The Quake Engine available at

http: //www.codeproject.com/managedcpp/quake2.asp
} 


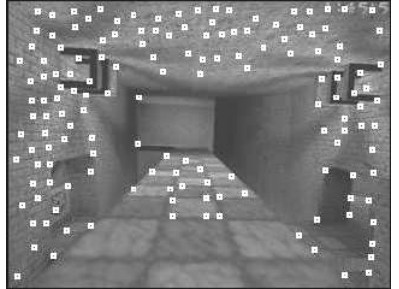

(a) Frame 1

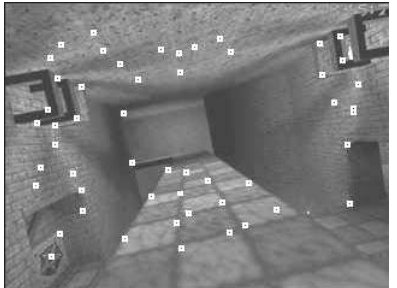

(b) Frame 5

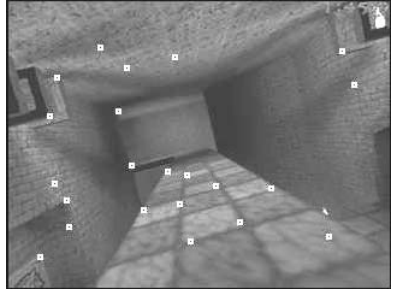

(c) Frame 10

Fig. 1. The "rolling sequence" with the feature points superimposed: (a) frame 1 (150 feature points); (b) frame 5; (c) frame 10 (22 feature points)

correspondences. In the sequences, we defined a forward translation, and the roll angles of the "observer" were altered according to the expression:

$$
\text { roll angles }(\mathrm{deg})=-6 \times \sin \left(2 \pi \times t_{f} / 30+1.1\right) \text {, }
$$

where $t_{f}$ is the image frame index.

In Phase 1, feature tracking shown in Fig. 1 leads to the history of the roll angles shown in Fig. 2 (a). This model is used for subsequent tracking with an interval of 3 frames. Fig. 2 (b) shows the 25 frames immediately succeeding the learning phase, in which our gait-based strategy clearly outperforms the STK scheme in terms of measurement accuracy. The longer interval between the neighbouring frames leads to the violation of the linearisation assumption in the STK scheme, resulting in large errors in the estimated motion parameters.

In further experiments, we also employ real data from a camera mounted on a pedestrian. As we do not have independent extraction of gait in this case, we compare texture mapped images using extracted parameters with the real image data, which gives a

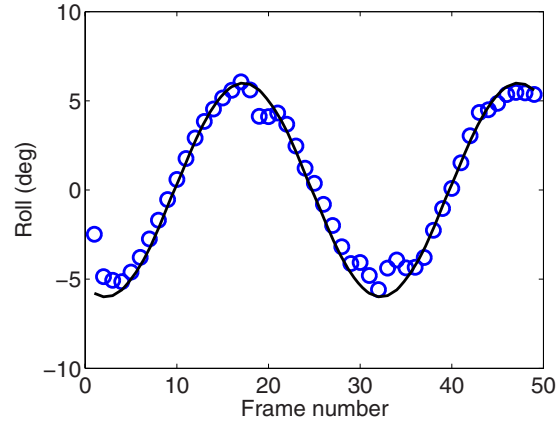

(a) Roll of frames 1-50

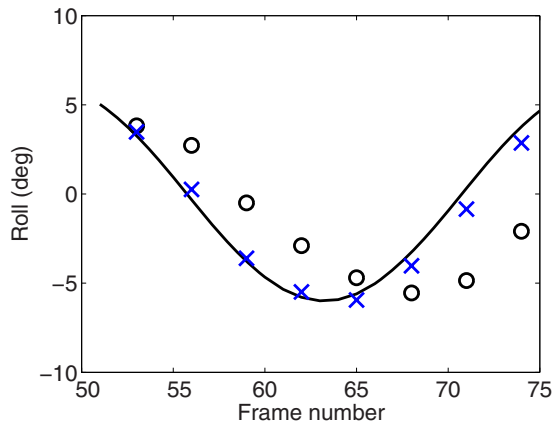

(b) Roll of frames 51-75

Fig. 2. Performance comparisons of the STK and the gait-based tracker: the line denotes the ground truth, the circles the result of the STK tracker, and the crosses that of our gait-based tracker 


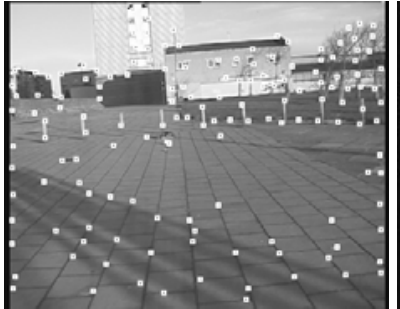

(a) 1 st frame

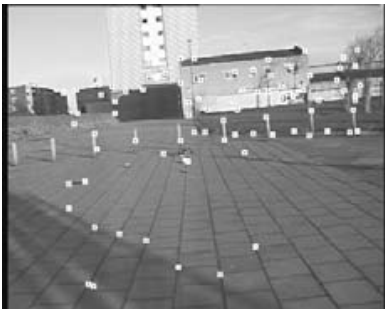

(b) 20th frame

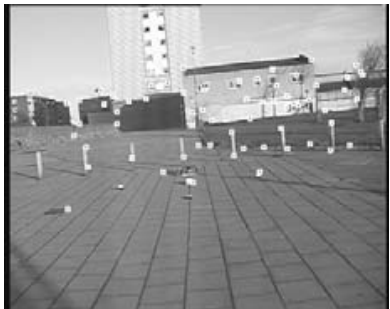

(c) 50th frame

Fig. 3. Real walking sequence with feature points superimposed

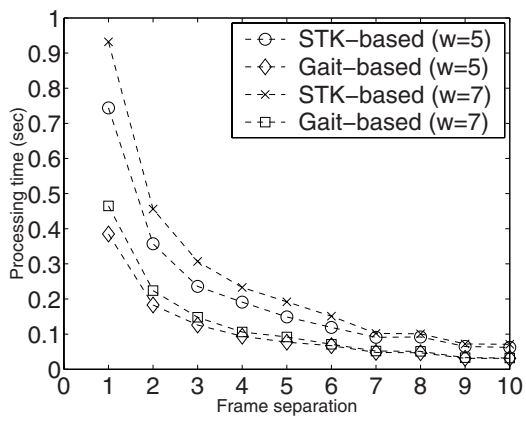

(a) Processing time

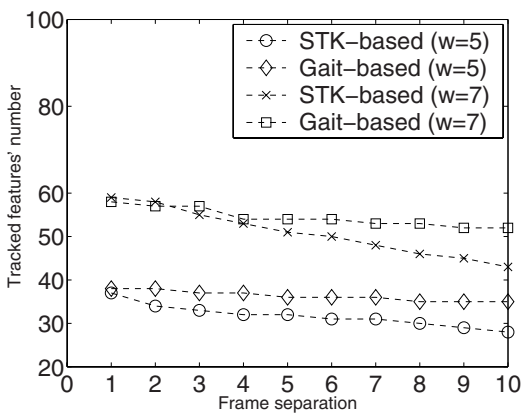

(b) Retention of features

Fig. 4. Comparison between the STK-based and gait-based framework in tracking real walking sequence $(w=$ width of search window)

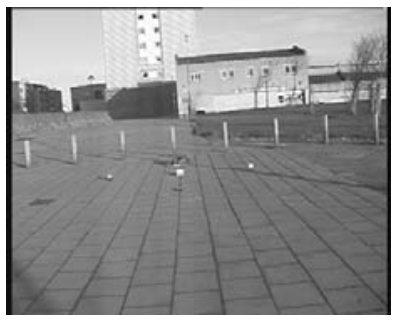

(a)

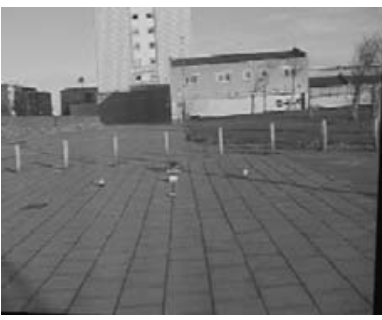

(b)

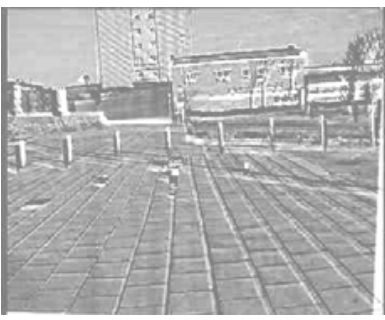

(c)

Fig. 5. Comparison of real frames and their texture-maps for real walking sequence: (a) The actual frame 55. (b) The texture map of frame 55 created by rendering. (c) Subtraction of (a) and (b). For better visibility, the brightness/contrast of (c) has been increased.

strong subjective comparison. Fig. 3 gives some example frames from an image sequence collected by the camera, where the detected and tracked feature points are superimposed. After a learning period of 50 image frames, we obtain a motion pattern. We then predict the motion parameters in successive frames, followed by immediate correction using the gait-based scheme. 
Fig. 4 shows a comparison between the STK- and gait-based approaches in tracking the sequence of Fig. 3 in terms of processing time (using a Pentium II-300 MMX PC), and retention of features. Compared to the STK-based approach using a fixed number of pyramid levels, our gait-based method localises the search better and hence reduces the time taken to find a match. The gait-based scheme also loses fewer feature points compared to the STK-based strategy.

In Fig. 5 we show the real and the texture mapped scenes for a frame using the estimated motion parameters. The subtraction image between the two demonstrates the accuracy of the approach.

\section{Conclusions and Future Work}

In this paper we have presented an approach for effective, efficient and robust egomotion tracking using a single mobile camera without using any other indicators of position, speed or inclination. An initial gait model is extracted from a fixed training period of two strides, using feature correspondences to estimate the ego-motion parameters, represented as a truncated Fourier series. Experiments on synthetic and real data have shown that the proposed strategy has more accurate and efficient ego-motion estimates and structural recovery than a comparable method that does not incorporate long-term motion estimates.

\section{References}

1. Oliensis, J.: A critique of structure-from-motion algorithms. Computer Vision and Image Understanding 80, 172-214 (2000)

2. Nava, F., Martel, A.: Wavelet modeling of contour deformations in Sobolev spaces for fitting and tracking applications. Pattern Recognition 36, 1119-1130 (2003)

3. Ristic, B., Arulampam, S., Gordon, N.: Beyond the Kalman Filter: Particle Filters for Tracking Applications. Artech House (2004)

4. Vidal, R., Hartley, R.: Three-view multibody structure from motion. IEEE Trans. Pattern Anal. Mach. Intell. 30, 214-227 (2008)

5. Molton, N., Brady, J.: Modelling the motion of a sensor attached to a walking person. Robotics and Autonomous Systems 34, 203-221 (2001)

6. Lepetit, V., Fua, P.: Monocular model-based 3D tracking of rigid objects: a survey. Foundations and Trends in Computer Graphics and Vision 1, 1-89 (2005)

7. Shi, J., Tomasi, C.: Good features to track. In: International Conference on Computer Vision and Pattern Recognition, pp. 593-600 (1994)

8. Smith, S., Brady, J.: SUSAN: A new approach to low-level image-processing. Int. J. of Comput. Vis. 23, 45-78 (1997)

9. Zhou, H., Green, P., Wallace, A.: Fundamental matrix estimation using generalized least squares. In: Proc. of International Conference on Visualisation, Imaging, and Image Processing, pp. 79-85 (2004)

10. Zhou, H.: Efficient motion tracking and obstacle detection using gait analysis. $\mathrm{PhD}$ thesis, Heriot-Watt University, Edinburgh, UK (2005)

11. Beaton, A., Tukey, J.: The fitting of power series, meaning polynormials, illustrated on bandspectroscopic data. Technometrics 16, 147-185 (1974) 